\title{
Trygve Haavelmo and the Emergence of Causal Calculus
}

\author{
Judea Pearl \\ University of California, Los Angeles \\ Computer Science Department \\ Los Angeles, CA, 90095-1596, USA \\ (310) 825-3243 \\ judea@cs.ucla.edu
}

\begin{abstract}
Haavelmo was the first to recognize the capacity of economic models to guide policies. This paper describes some of the barriers that Haavelmo's ideas have had (and still have) to overcome, and lays out a logical framework that has evolved from Haavelmo's insight and matured into a coherent and comprehensive account of the relationships between theory, data and policy questions. The mathematical tools that emerge from this framework now enable investigators to answer complex policy and counterfactual questions using simple routines, some by mere inspection of the model's structure. Several such problems are illustrated by examples, including misspecification tests, nonparametric identification, mediation analysis, and introspection. Finally, we observe that economists are largely unaware of the benefits that Haavelmo's ideas bestow upon them and, to close this gap, we identify concrete recent advances in causal analysis that economists can utilize in research and education.
\end{abstract}

\section{Introduction}

To students of causation, Haavelmo's paper "The statistical implications of a system of simultaneous equations," (Haavelmo, 1943) marks a pivotal turning point, not in the statistical implications of econometric models, as historians typically presume, but in their causal counterparts. Causal implications, which prior to Haavelmo's paper were cast to the mercy of speculation and intuitive judgment have thus begun their quest for full membership in the good company of scientific discourse.

Haavelmo introduced three revolutionary insights in 1943.

First, when an economist sits down to write a structural equation he/she envisions, not statistical relationships but a set of hypothetical experiments, qualitative aspects of which are then encoded in the system of equations. Second, an economic model thus constructed is capable of answering policy intervention questions, with no further assistance from the modeler. Finally, to demonstrate the feature above, Haavelmo presented a mathematical procedure that takes an arbitrary model and produces quantitative answers to policy questions (see Section 1.3).

\subsection{What is an economic model?}

This first idea, that an economic model depicts a series of hypothetical experiments was expressed more forcefully in Haavelmo's 1944 paper (The Probability Approach in Econometrics) where he states:

"What makes a piece of mathematical economics not only mathematics but also economics is, I believe, this: When we set up a system of theoretical relationships and use economic names for the otherwise purely theoretical variables involved, we have in mind 
some actual experiment, or some design of an experiment, which we could at least imagine arranging, in order to measure those quantities in real economic life that we think might obey the laws imposed on their theoretical namesakes." (1944, p. 5)

But the methodological implications of this idea is demonstrated more explicitly in 1943, where Haavelmo tries to explain what a modeller must have in mind in putting together two or more simultaneous equations, say

$$
\begin{aligned}
& y=a x+\epsilon_{1} \\
& x=b y+\epsilon_{2}
\end{aligned}
$$

Haavelmo first showed that, contrary to naive expectation, the term $a x$ is not equal to $E(Y \mid x)^{1}$ and, so, asked Haavelmo, what information did the modeller intend $a$ to carry in Eq. (1), and what information would $a$ provide if we are able to estimate its value.

In posing this question, Haavelmo addressed the dilemma of incremental model construction. Given that the statistical content of $a$ can only be discerned (if at all) by considering the entire system of equations, how can a modeller write down one equation at a time, without knowing what the meaning of the coefficients is in each equation. "What is then the significance of the theoretical equations..." Haavelmo asked (1943, p. 11) and answered it immediately: "To see that, let us consider, not a problem of passive predictions, but a problem of government planning."

In modern terms, Haavelmo rejected the then-ruling paradigm that parameters are conveyors of statistical information and prepared the ground for the causal definition of $a$ (Pearl, 1994):

$$
a=\frac{\partial}{\partial x} E(Y \mid d o(x))
$$

which refers to a controlled experiment in which an agent (e.g., Government) is controlling $x$ and observing $y{ }^{2}$ In such experiment, the average slope of $Y$ on $X$ (i.e., $a$ ) bears no relationship to the regression slope (i.e., $\frac{\partial}{\partial x} E(Y \mid X=x)$ ) in the population prior to intervention. Whereas the statistical content of $a$ (if identified) may come from many equations, its causal content is local - to the great relief of most economists who think causally, not statistically.

This simple truth, which today is taken (almost) for granted, took a long time to take roots. To illustrate, the fierce debate between prominent statisticians and economists that flared up in 1992, fifty years after Haavelmo's paper, revolved precisely around this issue of interpreting the meaning of $a$. The economist in the debate, Arthur Goldberger (1992), claimed that $a x$ in Eq. (1) may be interpreted as the expected value of $Y$ "if $x$ were fixed," so that the $a$ parameter "has natural meaning for the economist." The statistician, Nanny Wermuth (1992), argued that, since $a x \neq E(Y \mid X=x)$, "the parameters in (1) cannot have the meaning Arthur Goldberger claims they have." Summarizing their arguments, Wermuth concluded that structural coefficients have dubious meaning, and Goldberger retorted that statistics has dubious substance. Remarkably, each side quoted Haavelmo to prove the other wrong, and both sides were in fact correct; structural coefficients have no meaning in terms of properties of joint distribution functions, the only meaning that statisticians were willing to accept in the 1990's. And statistics has no substance, if it excludes from its province all aspects of the data generating mechanism that do not show up in the joint distribution, for example, $a$, or $E(Y \mid d o(x))$.

The confusion did not end in 1992. The idea that an economic model must contain extrastatistical information, that is, information that can not be derived from joint densities, and that the gap between the two can never be bridged, seems to be very slow in penetrating the mind set

\footnotetext{
${ }^{1}$ Although Haavelmo used nonrecursive models to get his point across, this inequality prevails in almost all economic models, certainly those in which $a$ is not identified.

${ }^{2}$ More precisely, the general definition of $a$ is $a=\frac{\partial}{\partial x}\left[Y_{x, z}(u)\right]$ where $Y_{x, z}(u)$ is the counterfactual " $Y$ if $x$ and $z$ " for unit $u$ (see Definition 1 and Appendix 1) and $Z$ is any set of variables in the model (excluding $X$ and $Y$ ). However, counterfactuals were rather late to obtain a formal representation in structural economics (Balke and Pearl, 1995; Heckman, 2000; Simon and Rescher, 1966). A simple recipe for computing $E(Y \mid d o(x))$ from any given model is given by Eq. (4) below, together with the identity $P(Y=y \mid d o(x))=P\left(Y_{x}=y\right)$. Note that it is only through the causal interpretation of $a$ that we can explain why an economist would exclude from Eq. (1) factors that are strong redactors of $Y$, yet are not deemed to be causes of $Y$.
} 
of mainstream economists. Hendry, for example, wrote: "The joint density is the basis: SEMs are merely an interpretation of that" (Hendry, 1998, personal communication). Spanos (2010), expressing similar sentiments, hopes to "bridge the gap between theory and data" through the teachings of Fisher, Neyman and Pearson, disregarding the fact that the gap between data and theory is fundamentally unbridgeable. This "data-first" school of economic research continues to pursue such hopes, unable to internalize the hard fact that statistics, however refined, cannot provide the causal information that economic models must encode to be of use to policy making. ${ }^{3}$

The dominance of statistical thinking in econometrics goes beyond theory testing. A highly influential econometric textbook writes: "A state implements tough new penalties on drunk drivers: What is the effect on highway fatalities?... [This effect] is an unknown characteristic of the population joint distribution of $X$ and $Y$ " (Stock and Watson, 2011, Ch. 4, p. 107). The fact that "effects" are not characteristics of population joint distributions, so compellingly demonstrated by Haavelmo (1943; see Eq. (1)-(3)), would probably come as a surprise to modern authors of econometric texts. To witness, almost seventy years after Haavelmo defined a model as a set of hypothetical experiments, the common definition of "Econometric Models" reads (Wikipedia, February 18, 2012): "An econometric model specifies the statistical relationship that is believed to hold between the various economic quantities pertaining to particular economic phenomena under study." ${ }^{4}$

\subsection{An oracle for policies or an aid to forecasters?}

Haavelmo's second and third insights also took time to be fully appreciated. Even today, the idea that an economic model should serve as an oracle (i.e., a provider of valid answers to non-trivial questions) for interventional questions tends to evoke immediate doubts and resistance: "How can one predict outcomes of experiments that were never performed, nor envisioned by the modeller?" Ask the skeptics. And if the modeller's assumptions possess such clairvoyant powers, why not ask the modeller to answer policy questions directly, rather than engage in modeling and analysis? How can a set of ordinary equations encapsulate the information needed for predicting the vast variety of interventions that a policy maker may wish to evaluate? How is this vast amount of information encoded nonparametrically, and what means do we have to extract it from its encoding? ${ }^{5}$

To a large extent, this typical resistance stems from the absence of distinct mathematical notation for marking the causal assumptions that enter into an economic model; the syntax of the equations appears deceptively algebraic, similar to that of regression models, hence void of causal content. Some economists, lured by this surface similarity, were led to conclude: "We must first emphasize that, disturbance terms being unobservable, the usual zero covariances "assumptions" generally reduce to mere definitions and have no necessary causality and exogeneity implications." (Richard, 1980, p. 3).

The absence of distinct notation for causal assumptions further compelled economists to assume that, to qualify for policy analysis, an economic model must be hardened by some extra ingredients; the equations themselves, even those ordained and causally interpreted by Haavelmo and the Cowles Commission, were deemed too simplistic or "fragile" to convey interventional information.

The literature on "exogeneity" (e.g. Engle, Hendry, and Richard, 1983; Hendry, 1995; Richard, 1980) for example, sought such extra power in the notion of "parameter invariance." Similarly, Cartwright (2007) views models as close to useless for policy evaluation because "the policy may affect a host of changes in other variables in the system, some envisaged and some not" (see Pearl

\footnotetext{
${ }^{3}$ Even the "faithfulness" assumption used in causal discovery algorithms (Pearl, 2000, Ch. 2; Pearl and Verma, 1991; Spirtes, Glymour, and Scheines, 1993) is extra statistical, for it cannot be tested from density functions over observed variables. This assumption, however, is milder than those made in structural equation modeling, for it is generic, and does not rely on problem-specific knowledge.

${ }^{4}$ I was tempted to correct this sentence in the Wikipedia, but decided to keep it as a witness to prevailing views, and as an incentive for editors of respected journals of econometrics to bring the issue to public discussion and collective revision.

${ }^{5}$ These rhetorical questions, which are rarely asked about physics or engineering, have repeatedly been posed to the author about economic modeling, reflecting the general reluctance of economists to examine the power of nonparametric equations (as in Section 3.2). Another recurrent question goes: "How do we establish those assumptions? Don't we sweep the most difficult issues under the rug when we agree to rely on them?" See footnote 11 and 12 for responses.
} 
2010d for rebuttal). And, in general, one would be hard pressed to find an economic textbook that encourages readers to answer policy questions from the equations themselves, without resorting to meta-mathematical disclaimers or preconditions that reside outside the model.

This lack of confidence in the ability of economic models to guide policies has threatened the utility of the entire enterprise of economic modeling for, taken to extreme, it commits economic analysis to statistical extrapolation of time series data. I doubt Haavelmo would agree to such restriction. Indeed, what is the point of parameter estimation if at the end of such exercise one must appeal to judgment to decide which parameter is invariant and which is not, or, lacking such judgment, to physically trying out the policy and observing its effect on various parameters.

A more reasonable alternative, one that I have advocated in (Pearl, 2000) and that is gaining support among economists (e.g., Heckman, 2000, 2003, 2008; Keane, 2010; Leamer, 2010) is to treat an economic model as an oracle for all causally related queries, including questions of prospective and introspective counterfactuals and, simultaneously, insist on encoding the assumptions needed for answering such queries within the model itself, not external to it. In other words, these assumptions should be guiding the modeller in the way the equations are authored. Moreover, even if the model is misspecified it can still be useful to policy makers, if each of its conclusion is accompanied by a meaningful set of assumptions, as long as as each assumption points to a condition that could conceivably be realizable or achievable.

"And what if an intervention changes the very equation that purports to predict its effect?" ask the critics and cite Lucas Jr. (1976), who attributed the predictive failure of macro-econometric models of the 1960s and 1970s to their non-invariance under changes of policy regime. What Lucas argued in fact was that, to get useful policy advice from a model we have to a) specify the model correctly and b) pose the right questions to it. Since the model provides the facility for encoding side effects associated with any given implementation of the policy evaluated, neglecting to encode them in the model constitutes a case of query misspecification, posing no lesser threats than model misspecification. In other words, if an intervention $I$, intended to increase variable $X$ from $X=x$ to $X=x^{\prime}$, has a side effect on some other variables or parameters, it would be inappropriate to seek the estimation of $P\left(y \mid d o\left(x^{\prime}\right)\right)$; the proper query should be the estimation of $P(y \mid d o(I))$, so as to take into account the various side effects of $I .{ }^{6}$ The burden of properly specifying queries rests with the query provider not with the model.

\subsection{The algorithmization of interventions}

Modern days interest in causal models and their tentative conclusions, owes its renaissance to Haavelmo's third insight - a concrete procedure for eliciting answers to policy questions from the model equations. This he devised at the end of his 1943 paper:

"Assume that the Government decides, through public spending, taxation, etc., to keep income, $r_{t}$, at a given level, and that consumption $u_{i}$ and private investment $v_{i}$ continue to be given by (2.5) and (2.6), the only change in the system being that, instead of (2.7), we now have

$$
r_{i}=u_{i}+v_{i}+g_{i}
$$

where $g_{i}$ is Government expenditure, so adjusted as to keep $r$ constant, whatever be $u$ and $v, \ldots "(1943$, p. 12)

This idea of simulating an intervention on a variable by modifying the equation that determines that variable while keeping all other equations intact is the basis of all currently used formalisms of causal inference. Haavelmo's proposal of adding an adjustable term to the equation so as to keep the manipulated variable constant differs somewhat from Fisher's proposal of subjecting such a variable to randomized external variations. Haavelmo was more interested in simulating the actual

\footnotetext{
${ }^{6}$ Cartwright's 2007 used the term "impostor counterfactuals" to describe the consequences of substituting compound interventions (e.g., do(I)) with atomic interventions (e.g., do(x)) (see (Hoover, 2011; Pearl, 2010d)). Compound interventions are analyzed by computing the simultaneous effects of their atomic components (Pearl, 2000, Ch. 4), which may consist of mild or drastic changes in the equations themselves (Pearl, 2000, Section 3.2.3).
} 
implementation of a pending policy, rather than the Fisherian experiment from which we may learn about the average effect of the policy.

Haavelmo's approach was later transformed by Strotz and Wold (1960) into the operation of "wiping out" the equation altogether, and was further translated into graphical models as "wiping out" incoming arrows into the manipulated variable (Pearl, 1993; Spirtes et al., 1993). ${ }^{7}$ This operation, called do-operator, has subsequently led to do-calculus (Pearl, 1994, 2000) and to the structural theory of counterfactuals (Balke and Pearl, 1995; Pearl, 2000, Ch. 7), which unifies structural equation modeling with the potential outcome paradigm of Neyman (1923) and Rubin (1974) and the possible-world semantics of Lewis (1973).

Key to this unifying framework has been a symbolic procedure for reading counterfactual information in a system of economic equations, as articulated in the following Definition:

Definition 1 (unit-level counterfactuals) (Pearl, 2000, p. 98)

Let $M$ be a fully specified structural model and $X$ and $Y$ two arbitrary sets of variables in $M$. Let $M_{x}$ be a modified version of $M$, with the equation(s) of $X$ replaced by $X=x$ (see Fig. 2(b), Section 3.1). Denote the solution for $Y$ in the modified model by the symbol $Y_{M_{x}}(u)$, where $u$ stands for the values that the exogenous variables take for any given individual (or unit) in the population. The counterfactual $Y_{x}(u)$ (Read: "The value of $Y$ in unit $u$, had $X$ been $x$ ") is defined by

$$
Y_{x}(u) \triangleq Y_{M_{x}}(u) .
$$

In words: The counterfactual $Y_{x}(u)$ in model $M$ is defined by the solution for $Y$ in the modified submodel $M_{x}$, with the exogenous variables held at $U=u$. For example, in Haavelmo's model of equations (1)-(2), the modified model $M_{x}(u)$ consists of Eq. (1) alone, with $x$ treated as a constant. The counterfactual $Y_{x}(u)$ therefore becomes $a x+\epsilon_{1}(u)$, with $\epsilon_{1}(u)$ standing for the omitted factors that characterize unit $U=u .^{8}$

We see that every structural equation, say $y=a x+\epsilon_{1}(u)$ (Eq. (1)), carries counterfactual information, $Y_{x}(u)=a x+\epsilon_{1}(u)$, which, in our simple case, conveys the assumptions of effect-linearity and effect homogeneity (i.e., $Y_{x}(u)-Y_{x^{\prime}}(u)=a\left(x-x^{\prime}\right)$, for all $u$ ). The structural assumption is in fact much stronger. The fact that the equation contains only $X$ on the right hand side conveys the counterfactual assumption (known as an "exclusion restriction") $Y_{x z}(u)=a x+\epsilon_{1}(u)$, where $Z$ is any set of variables (in the model) that does not appear on the right hand side of the equation. The exclusion restriction and linearity assumption are refutable in interventional experiments, not so the homogeneity assumption. ${ }^{9}$ Naturally, when the exogenous variables $U$ in a model are random variables, the counterfactual $Y_{x}$ will be a random variable as well, the distribution of which is dictated by both the distribution $P(U=u)$ of the exogenous variables and the structure of the model $M_{x}$. This interpretation permits us to define joint distributions of counterfactual variables and to detect conditional independencies of counterfactuals directly from the structure of the model (Pearl, 2000, Ch. 7).

Equation (4) constitutes the bridge between the structural interpretation of counterfactuals and the potential outcome framework advanced by Neyman (1923) and Rubin (1974), which takes the controlled randomized experiment as its guiding paradigm (see Appendix 1). One of the main differences between the two frameworks is that counterfactuals, as well as assumptions such as "ignorability," "sequential ignorability," or "instrumentality" can actually be derived from the economic model (see Appendix 1); they need not be imposed as separate assumptions external to, and oblivious to the model. Another difference is that the antecedent $x$ in the structural interpretation of

\footnotetext{
${ }^{7}$ Figure 2(b) (Sectioin 3.1) provides a graphical representation of the model that results from Haavelmo's intervention. Some authors prefer to retain those arrows in the graph and split outgoing arrows instead (Heckman and Pinto, 2013); the resulting equations and all their implications are the same (Pearl, 2013b).

${ }^{8}$ The set of units characterized by the same values $U=u$ of the exogenous variables form an equivalent class. We therefore do not distinguish between "unit" as an index for individual identity and "unit" as a specific instantiation $U=u$ of the exogenous variables.

${ }^{9}$ Anecdotically, none of the six textbooks surveyed in (Chen and Pearl, 2013) explains to readers what justification there is for excluding variables from an equation; such explanations require that equations be given causal interpretation, which textbooks are reluctant to do.
} 
$Y_{x}(u)$ need not be a manipulable treatment but may consist of any exogenous or endogenous variable (e.g., sex, genetic traits, race, earning) that affects $Y$ as part of a social or biological process (Heckman, 2008). This interpretation has extended Haavelmo's theory of interventions from linear to nonparametric analysis and permitted questions of identification, estimation, and generalization to be handled with mathematical precision and algorithmic simplicity (see Section 3).

Haavelmo did not deem his intervention theory to be revolutionary, but natural. In his words:

"That is, to predict consumption ... under the Government policy,... we may use the 'theoretical' equations obtained by omitting the error terms..."

"this is only natural, because now the Government is, in fact, performing 'experiments' of the type we had in mind when constructing each of the two equations." (1943, p. 12)

I do consider it revolutionary in that it defines the effect of interventions not in terms of the model's parameters but in terms of a procedure (or "surgery") that hypothetically modifies the structure of the model so as to simulate the actual intervention. ${ }^{10}$ It thus liberates economic analysis from its dependence on parametric representations and permits a totally nonparametric calculus of causes and counterfactuals that makes the connection between assumptions and conclusions explicit and transparent.

In the next section I will give a brief summary of nonparametric structural models and the wealth of mathematical tools that they now offer to economists and other policy-minded data analysts.

\section{The Logic of Structural Causal Models (SCM)}

This section describes a coherent theory of causal inference that I propose to call Structural Causal Model (SCM). It takes seriously the original insights of Haavelmo and the subsequent philosophy of the Cowles Commission program and, enriched with a few ideas from logic and graph theory, provides a unifying framework for all known approaches to causation.

A simple way to view SCM is to imagine a logical machine, or an inference engine, ${ }^{11}$ that takes three inputs and produces three outputs. The inputs are:

I-1. A set $A$ of qualitative causal assumptions that the investigator is prepared to defend on scientific grounds, and a model $M_{A}$ that encodes these assumptions. Traditionally, $M_{A}$ takes the form of a set of structural equations with undetermined parameters. A typical assumption is that certain omitted factors, represented by error terms, are uncorrelated, or that no direct effect exists between a pair of variables (i.e., an "exclusion restriction").

I-2. A set $Q$ of queries concerning causal and counterfactual relationships among variables of interest. Traditionally, $Q$ concerned the magnitudes of structural parameters but, in general, $Q$ may address causal relations more directly, e.g.,

$Q_{1}$ : What is the effect of treatment $X$ on outcome $Y$ ?

$Q_{2}$ : Is this employer guilty of gender discrimination?

Formally, each query $Q_{i} \in Q$ should be computable from a fully specified theoretical model $M$ in which all functional relationships are given, together with the joint distribution of all omitted factors. Non-computable queries are inadmissible.

\footnotetext{
${ }^{10}$ Fearing violation of modularity, Cartwright (2007) and Heckman and Vytlacil (2007) voiced objections to hypothetical modifications of the model's equations as proposed by Haavelmo. These objections are addressed in (Pearl, 2009a, pp. 362-265, 374-380), with emphasis on the fundamental distinctions between definition, identification, estimation, and implementation, which become crisp and unambiguous in nonparametric structural causal models (Section 2).

${ }^{11}$ These terms are chosen to emphasize that, in dealing with econometric modeling, it is essential to separate the logic of the method from the veracity of its premises. Surely, the long term goal of economics is to see every premise substantiated by compelling empirical evidence, and the importance of efforts to establish such evidence from sources residing outside the model is far from being overlooked by this author. However, in any given study, including those evidence-seeking efforts, the aim is to take what little theoretical knowledge we have, and make sure it is maximally utilized, while acknowledging its provisional status.
} 
I-3. A set $D$ of experimental or non-experimental data.

The outputs are

O-1. A set $A^{*}$ of statements which are the logical implications of $A$, prior to obtaining any data. For example, that $X$ has no effect on $Y$ if we hold $Z$ constant, or that $Z$ is an instrument relative to a pair $\{X, Y\}$.

O-2. A set $C$ of data-dependent claims (or conclusions) concerning the magnitudes or likelihoods of the target queries in $Q$, each conditional of $A$. $C$ may contain, in the simple case, the estimated mean and variance of a given structural parameter, or the expected effect of a given intervention or, to illustrate a counterfactual query, the probability that a student trained in a given program who now earns $50 \mathrm{~K}$ per year would not have reached a salary level gre ater than 30K had he/she not been trained (Pearl, 2000, Ch. 9).

Auxiliary to $C$, SCM also generates an estimand $Q_{i}(P)$ for each query in $Q$, or a determination that $Q_{i}$ is not identifiable from $P$, the joint density of observed variables.

O-3. A list $T$ of testable statistical implications of $A$, and the degree $g\left(T_{i}\right), T_{i} \in T$, to which the data agrees with each of those implications. A typical implication would be the vanishing of a specific regression coefficient, or the invariance of such coefficient to the addition or removal of a given regressor; such constraints can be read from the model $M_{A}$ and confirmed quantitatively by the data.

The structure of this inferential exercise is shown schematically in Fig. 1.

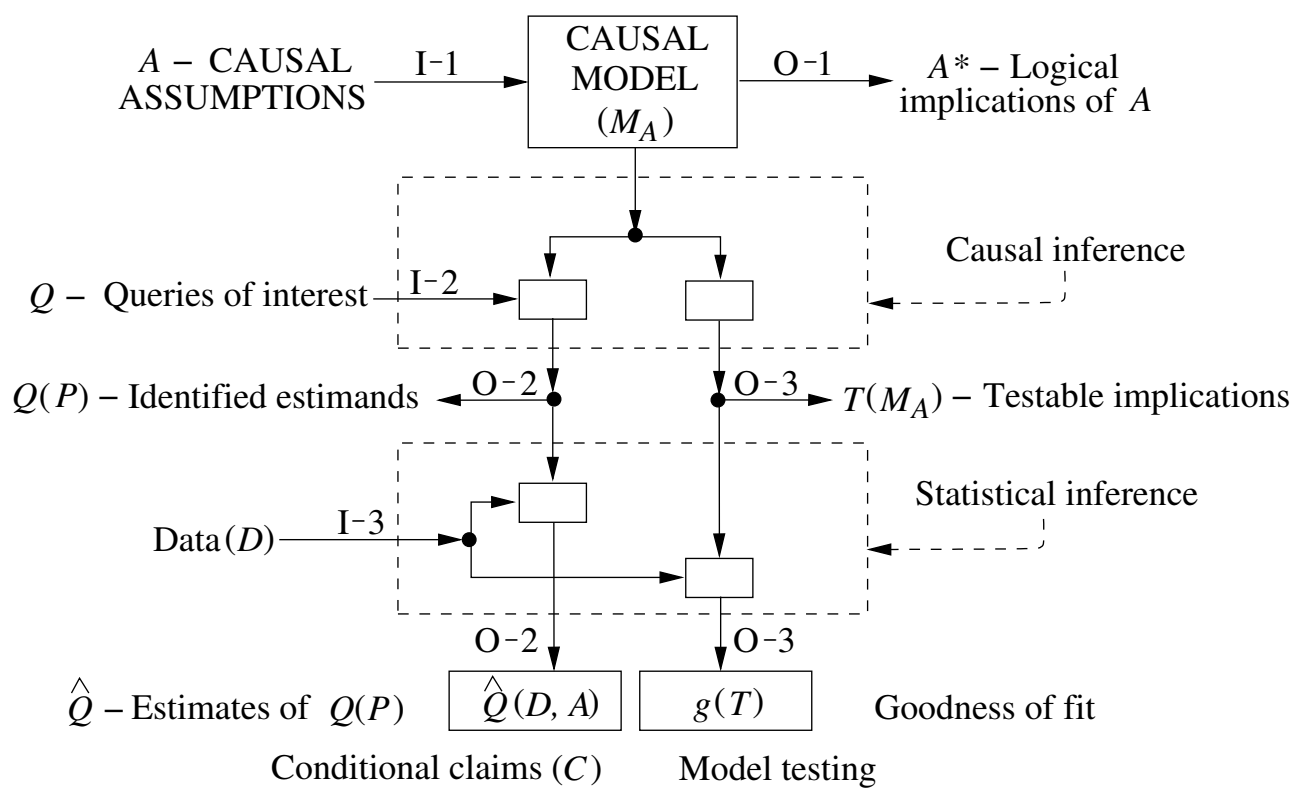

Figure 1: SCM methodology depicted as the an inference engine converting assumptions $(A)$, queries $(Q)$, and data $(D)$ into logical implications $\left(A^{*}\right)$ Conditional claims $(C)$ and data-fitness indices $(g(T))$.

Several observations are worth noting before illustrating these inferences by examples. First, SCM is not a traditional statistical methodology, typified by hypothesis testing or estimation, because neither claims nor assumptions are expressed in terms of probability functions of realizable variables (Pearl, 2000).

Second, all claims produced by SCM are conditional on the validity of $A$, and should be reported in conditional format: "If $A$ then $C_{i}$ " for any claim $C_{i} \in C$. Such claims assert that anyone willing to accept $A$, must also accept $C_{i}$ out of logical necessity. Moreover, no other method can do better, 
that is, if SCM analysis finds that a subset $A^{\prime}$ of assumptions is necessary for inferring a claim $C_{i}$, no other methodology can infer $C_{i}$ with a weaker set of assumptions. This follows from casting the relationship between $A$ and $C$ in a formal mathematical system, coupled with the completeness theorems of Halpern (1998) and Shpitser and Pearl (2008). ${ }^{12}$

Thirdly, passing a goodness-of-fit test is not a prerequisite for the validity of the conditional claim "If $A$ then $C_{i}$," nor for the validity of $C_{i}$. While it is important to know if any assumptions in $A$ are inconsistent with the data, $M_{A}$ may not have any testable implications whatsoever. In such a case (traditionally called "just identified"), the assertion "If $A$ then $C_{i}$ " may still be extremely informative in a decision making context, since each $C_{i}$ conveys quantitative information extracted from the data compared with the qualitative assumptions $A$ with which the study commences. Moreover, even if $A$ turns out inconsistent with $D$, the inconsistencies may be entirely due to portions of the model which have nothing to do with the derivation of $C_{i}$ (Marschak, 1953). It is therefore important to identify which statistical implication of $A$ is responsible for the inconsistency; while global tests for goodness-of-fit hide this information, a variety of local tests have been developed as more viable alternatives (Pearl 2000, pp. 144-45; 2004).

Finally, and this has been realized by many researchers in the 1980's, there is nothing in SCM's methodology to protect $C$ from the inevitability of contradictory equivalent models, namely, models that satisfy all the testable implications of $M_{A}$ and still advertise claims that contradict $C$ (see footnote 19). Modern developments in graphical modeling have devised visual and algorithmic tools for detecting, displaying, and enumerating these equivalent models (Kyono, 2010). Researchers should keep in mind therefore that only a tiny portion of the assumptions behind each SCM lends itself to scrutiny by the data; the bulk of it must remain untestable, substantiated by scientific theories, controlled experiments, or conclusions of causal discovery algorithms (Pearl, 2000, Ch. 2; Pearl and Verma, 1991; Spirtes et al., 1993).

It is also important to emphasize that the inferential tools provided by SCM cannot be replaced or evaded by appealing to so called "alternative approaches" to causation, or to "causal pluralism" (Cartwright, 2007). The abilities (1) to articulate assumptions formally and transparently, (2) to decide if they permit identification and (3) to detect whether they have testable implications are three inescapable components of any "approach" that claims to guide policy. ${ }^{13}$

\section{Causal Calculus, Tools, and Frills}

By "causal calculus" I mean mathematical machinery for performing the computational tasks described in the inference engine of Fig. 1.

These include:

1. Tools of reading and explicating the causal assumptions embodied in structural models as well as the set of assumptions that support each individual causal claim.

2. Methods of identifying the testable implications (if any) of the assumptions encoded in the model, and ways of testing, not the model in its entirety, but the testable implications of the assumptions behind each causal claim.

3. Methods of deciding, prior to taking any data, what measurements ought to be taken, whether one set of measurements is as good as to another, and which adjustments need to be made so as to render our estimates of the target quantities unbiased.

\footnotetext{
${ }^{12}$ This is important to emphasize in view of often heard critics that, in SCM, one must start with a model in which all causal relations are presumed known, at least qualitatively. This is not so. It is common to start with a model in which no causal relation is assumed known, and ask "what must be ascertained in order to answer the research question at hand?" Additionally, if some causal assumptions in the model are found necessary, no other method can get away with weaker assumptions, though some tend to hide the assumptions under catch-all terms such as "ignorability," "as if randomized," "exchangeability," "quasi-experiment," "exogeneity," and the like.

${ }^{13}$ Remarkably, none of these components is currently taught in econometric classes (Chen and Pearl, 2013), and none is known to mainstream econometric researchers.
} 
4. Methods for devising critical statistical tests by which two competing theories can be distinguished.

5. Methods of deciding mathematically if the causal relationships of interest are estimable from non-experimental data and, if not, what additional assumptions, measurements or experiments would render them estimable.

6. Methods of recognizing and generating equivalent models.

7. Methods of locating instrumental variables for any relationship in a model, or turning variables into instruments when none exists (Brito and Pearl, 2002).

8. Methods of evaluating "causes of effects" and predicting effects of choices that differ from the ones actually made, as well as the effects of dynamic policies which respond to time-varying observations.

9. A solution to the so-called "Mediation Problem," which estimates the degree to which specific mechanisms contribute to the transmission of a given effect, in models containing both continuous and categorical variables, linear as well as nonlinear interactions (Pearl, 2001, 2012b).

10. A principled treatment of the problem of "external validity" (Campbell and Stanley, 1963), including, formal methods of deciding if a causal relation estimated in one population can be transported to another population, in which experimental conditions are different (Pearl and Bareinboim, 2011).

A full description of these techniques is given in (Pearl, 2000) as well as in recent survey papers (Pearl, 2010a,b). Here I will demonstrate by examples how some of the simple tasks listed above are handled in the nonparametric framework of a SCM.

\subsection{Two models for discussion}

Consider a nonparametric structural model defined over a set of endogenous variables $\left\{Y, X, Z_{1}\right.$, $\left.Z_{2}, Z_{3}, W_{1}, W_{2}, W_{3}\right\}$, and unobserved exogenous variables $\left\{U, U^{\prime}, U_{1}, U_{2}, U_{3}, U_{1}^{\prime}, U_{2}^{\prime}, U_{2}^{\prime}, U_{3}^{\prime}\right\}$. The equations are assumed to be structured as follows:

Model 1

$$
\begin{aligned}
Y & =f\left(W_{3}, Z_{3}, W_{2}, U\right) & X & =g\left(W_{1}, Z_{3}, U^{\prime}\right) \\
W_{3} & =g_{3}\left(X, U_{3}^{\prime}\right) & W_{1} & =g_{1}\left(Z_{1}, U_{1}^{\prime}\right) \\
Z_{3} & =f_{3}\left(Z_{1}, Z_{2}, U_{3}\right) & Z_{1} & =f_{1}\left(U_{1}\right) \\
W_{2} & =g_{2}\left(Z_{2}, U_{2}^{\prime}\right) & Z_{2} & =f_{2}\left(U_{2}\right)
\end{aligned}
$$

$f, g, f_{1}, f_{2}, f_{3}, g_{1}, g_{2}, g_{3}$ are arbitrary, unknown functions, and all exogenous variables are mutually independent but otherwise arbitrarily distributed.

For the purpose of our illustration, we will avoid assigning any economic meaning to the variables and functions involved, thus focusing on the formal aspects of such models rather than their substance. The model conveys two types of theoretical (or causal) assumptions:

1. Exclusion restrictions, depicted by the absence of certain variables from the arguments of certain functions, and

2. Causal Markov conditions, depicted by the absence of common $U$-terms in any two functions, and the assumption of mutual independence among the $U^{\prime}$ s.

Given the qualitative nature of these assumptions, the algebraic representation is superfluous and can be replaced, without loss of information, with the diagram depicted in Fig. 2(a). ${ }^{14}$ To anchor the discussion in familiar grounds, we also present the linear version of Model 1:

\footnotetext{
${ }^{14}$ This is entirely optional; readers comfortable with algebraic representations are invited to stay in their comfort zone.
} 


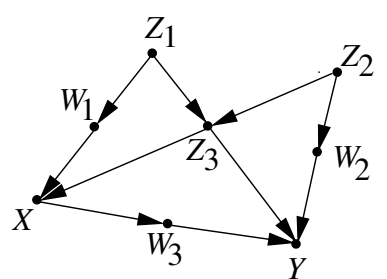

(a)

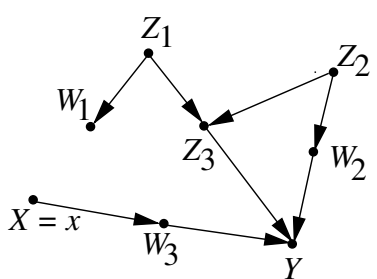

(b)

Figure 2: (a) A graphical representation of Model 1. Error terms are assumed mutually independent and not shown explicitly. (b) A graphical representation of Haavelmo's hypothetical model $M_{x}$ under the policy $d o(X=x)$.

Model 2 (Linear version of Model 1)

$$
\begin{array}{rlrl}
Y & =a W_{3}+b Z_{3}+c W_{2}+U & X & =t_{1} W_{1}+t_{2} Z_{3}+U^{\prime} \\
W_{3} & =c_{3} X+U_{3}^{\prime} & W_{1} & =a_{1}^{\prime} Z_{1}+U_{1}^{\prime} \\
Z_{3} & =a_{3} Z_{1}+b_{3} Z_{2}+U_{3} & Z_{1} & =U_{1} \\
W_{2} & =c_{2} Z_{2}+U_{2}^{\prime} & Z_{2} & =U_{2}
\end{array}
$$

All $U^{\prime} s$ are assumed to be uncorrelated.

In our case, the recursive nature of the equations of Model 1 results in a Directed Acyclic Graph (DAG), a structure that will be assumed throughout this paper. The basic principles of Havvelmo's intervention (e.g., Definition 1) are also applicable to systems with simultaneous equations (reciprocal causation), represented to cyclic graphs, though some of the computational tasks become more involved. While the orthogonality assumption renders these equations regressional, we can easily illustrate non-regressional models by assuming that some of the variables are not measurable.

\subsection{Illustrating typical question-answering tasks}

Given the model defined above, the following are typical questions that an economist may wish to ask.

\subsubsection{Testable implications (misspecification tests)}

a. What are the testable implications of the assumptions embedded in Model 1?

b. Assume that only variables $X, Y, Z_{3}$, and $W_{3}$ are measured, are there any testable implications?

c. The same, but assuming only variables $X, Y$, and $Z_{3}$ are measured,

d. The same, assuming all but $Z_{3}$ are measured.

e. Assume that an alternative model, competing with Model 1, has the same structure, with the $Z_{3} \rightarrow X$ arrow reversed. What statistical test would distinguish between the two models?

f. What regression coefficient in Model 2 would reflect the test devised in (e)?

\subsubsection{Equivalent models}

a. Which arrows in Fig. 2(a) can be reversed without being detected by any statistical test?

b. Is there an equivalent model (statistically indistinguishable) in which $Z_{3}$ is a mediator between $X$ and $Y$ (i.e., the arrow $X \leftarrow Z_{3}$ is reversed)? 


\subsubsection{Identification}

a. Suppose we wish to estimate the average causal effect of $X$ on $Y$

$$
A C E=P(Y=y \mid d o(X=1))-P(Y=y \mid d o(X=0)) .
$$

Which subsets of variables need to be adjusted to obtain an unbiased estimate of ACE?

[Recall: $P(Y=y \mid d o(X=1))$ is equal to the probability of $Y=y$ in the model of Fig. 2(b), under $X=1$.]

b. Is there a single variable that, if measured, would allow an unbiased estimate of ACE?

c. Assume we have a choice between measuring $\left\{Z_{3}, Z_{1}\right\}$ or $\left\{Z_{3}, Z_{2}\right\}$, which would be preferred?

\subsubsection{Instrumental variables}

a. Is there an instrumental variable for the $Z_{3} \rightarrow Y$ relationship?

If so, what would be the IV estimand for parameter $b$ in Model 2 ?

b. Is there an instrument for the $X \rightarrow Y$ relationship?

If so, what would be the IV estimand for the product $c_{3} c$ in Model 2?

\subsubsection{Mediation}

a. What variables must be measured if we wish to estimate the direct effect of $Z_{3}$ on $Y$ ?

b. What variables must be measured if we wish to estimate the indirect effect of $Z_{3}$ on $Y$, mediated by $X$ ?

c. What is the estimand of the indirect effect in (b), assuming that all variables are binary?

\subsubsection{Sampling selection bias ${ }^{15}$}

Suppose our aim is to estimate the conditional expectation $E(Y \mid X=x)$, and samples are preferentially selected to the dataset depending on a set $V_{S}$ of variables,

a. Let $V_{S}=\left\{W_{1}, W_{2}\right\}$, what set, $T$, of variables need be measured to correct for selection bias? (Assuming we can estimate $P(T=t)$ from external sources e.g., census data.)

b. In general, for which sets, $V_{S}$, would selection bias be correctable.

c. Repeat (a) and (b) assuming that our aim is to estimate the causal effect of $X$ on $Y$.

\subsubsection{Linear digressions}

Consider the linear version of our model (Model 2)

Question 1: Name three testable implications of this model

Question 2: Suppose $X, Y$, and $W_{3}$ are the only variables that can be observed. Which parameters can be identified from the data?

Question 3: If we regress $Z_{1}$ on all other variables in the model, which regression coefficient will be zero?

Question 4: If we regress $Z_{1}$ on all the other variables in the model and then remove $Z_{3}$ from the regressor set, which coefficient will not change?

\footnotetext{
${ }^{15}$ This section illustrates nonparametric extensions of Heckman's approach to selection bias (Heckman, 1979). A complete theory can be found in Bareinboim and Pearl (2012) and Bareinboim et al. (2014).
} 
Question 5: ("Robustness" - a more general version of Question 4.) Model 2 implies that certain regression coefficients will remain invariant when an additional variable is added as a regressor. Identify five such coefficients with their added regressors. ${ }^{16}$

\subsubsection{Counterfactual reasoning}

a. Find a set $S$ of endogenous variables such that $X$ would be independent of the counterfactual $Y_{x}$ conditioned on $S$.

b. Determine if $X$ is independent of the counterfactual $Y_{x}$ conditioned on all the other endogenous variables.

c. Determine if $X$ is independent of the counterfactual $W_{3, x}$ conditioned on all the other endogenous variables.

d. Determine if the counterfactual relationship $P\left(Y_{x} \mid X=x^{\prime}\right)$ is identifiable, assuming that only $X, Y$, and $W_{3}$ are observed.

\subsection{Solutions}

The problems posed in Section 3.2 read like homework problems in Economics 101 class. They should be! Because they are fundamental, easily solvable, and absolutely necessary for even the most elementary exercises in nonparametric analysis. Readers should be pleased to know that with the graphical techniques available today, these questions can generally be answered by a quick glance at the graph of Fig. 2 (see, for example, Greenland and Pearl (2011), Kyono (2010), or Pearl (2010a,b, 2012a)).

More elaborate problems, like those involving transportability or counterfactual queries may require the inferential machinery of $d o$-calculus or counterfactual logic. Still, such problems have been mathematized, and are no longer at the mercy of unaided intuition, as they are presented for example in Campbell and Stanley (1963).

It should also be noted that, with the exception of our linear digression (3.2.7) into Model 2, all queries were addressed to a purely nonparametric model and, despite the fact that the form of our equations and the distribution of the $U$ 's are totally arbitrary, we were able to extract answers to policy-relevant questions in a form that is estimable from the data available.

For example, the answer to the first identification question (a) is: The set $\left\{W_{1}, Z_{3}\right\}$ is sufficient for adjustment and the resulting estimand is:

$$
P(Y=y \mid d o(X=x))=\sum_{w_{1}, z_{3}} P\left(Y=y \mid X=x, Z_{3}=z_{3}, W_{1}=w_{1}\right) P\left(Z_{3}=z_{3}, W_{1}=w_{1}\right) .
$$

This can be derived algebraically using the rules of do-calculus or seen directly from the graph, using the back-door criterion (Pearl, 1993), which has become an indispensable tool for confounding control in epidemiology (Glymour and Greenland, 2008; Vansteelandt and Lange, 2012) and social science (Morgan and Winship, 2007). When a policy question is not identifiable, graphical methods can detect it and exit with failure. Put in econometric vocabulary, these results mean that the identification problem in nonparametric triangular simultaneous equations models is now solved. Given any such model, an effective algorithm exists that decides if the causal effect of any subset of variables on another is identifiable and, if so, the algorithm delivers the correct estimand (Shpitser and Pearl, 2008).

\footnotetext{
${ }^{16}$ According to White and Lu (2014) "A common exercise in empirical studies is a 'robustness check,' where the researcher examines how certain 'core' regression coefficient estimates behave when the regression specification is modified by adding or removing regressors." "of the 98 papers published in The American Economic Review during 2009, 76 involve some data analysis. Of these, 23 perform a robustness check along the lines just described, using a variety of estimators." Oster (2013) finds that $75 \%$ of 2012 papers published in The American Economic Review, Journal of Political Economy, and Quarterly Journal of Economics sensitivity to added regressors as indicative of misspecification. Since this practice is conducted to help diagnose misspecification, the answer to Question 5 is essential for discerning whether an altered coefficient indicates misspecification or not.
} 
The nonparametric nature of these exercises represents the ultimate realization of what Heckman calls the Marschak's Maxim (Heckman, 2010), referring to an observation made by Jacob Marschak (1953) that many policy questions do not require the estimation of each and every parameter in the system - a combination of parameters is all that is necessary and, moreover, it is often possible to identify the desired combination without identifying the individual components. The exercises presented above show that Marschak Maxim goes even further - the desired quantity can often be identified without ever specifying the functional or distributional forms of these economic models.

This nonparametric generality does not mean of course that graphical methods cannot accommodate stronger assumptions on the functions in the model, such as linearity, homogeneity, monotonicity or separability. For example, DAGs have provided critical insights into the behavior of linear causal systems (Pearl, 2013a). The most powerful identification results in linear econometric models have recently been derived using DAGs (Brito and Pearl, 2002; Foygel, Draisma, and Driton, 2012). The use of instrumental variables, which some authors refer to as "The Roy model" (Heckman and Pinto, 2013) has been extended substantially in both acyclic (Brito and Pearl, 2006) and cyclic (Phiromswad and Hoover, 2013) models. The instrumental inequality (Pearl, 2009a, p. 279) and tight bounds on the binary Roy Model (Balke and Pearl, 1997) were derived through DAG's representations. Finally, mediation and moderation effects in nonlinear parametric systems (Pearl, 2014) and attribution problems in monotonic systems (Pearl, 2009a, Ch. 9) are examples of specific identification constraints incorporated within the graphical model framework.

\subsection{What kept the Cowles Commission at bay?}

A natural question to ask is why these recent developments have escaped the attention of Marschak and the Cowles Commission who, around 1950, already adopted Haavelmo interpretation of structural models, and have formulated mathematically many of the key concepts and underlying theories that render structural models useful for policy making, including theories of identification, structural invariance and structural estimation. What then prevented them from making the next logical move and tackle nonparametric models such as those exemplified in Section 3.2?

I believe the answer lies in two ingredients that where not available to Cowles Commission's researchers and which are necessary for solving nonparametric problems. (These had to wait for the 1980-90's to be developed.) I will summarize these ingredients as "principles" since the entire set of tools needed for solving these problems emanate from these two:

Principle 1: "The law of structural counterfactuals."

Principle 2: "The law of structural independence."

The first principle is described in Definition 1 and instructs us how to compute counterfactuals from an economic model $M$. Simon and Rescher (1966) came close to this definition but, lacking the "wiping out" operator, could not reconcile the contradiction that evolves when an observation $X=x^{\prime}$ clashes with the antecedent $X=x$ of the counterfactual $Y_{x}$. Later economists, like Roy and Quandt, although they used counterfactual reasoning in their writings (Heckman, 2008), lacked the syntactic machinery for reading counterfactuals from a model, and could not therefore develop the tools necessary for solving the problems presented in Sections 3.2.3, 3.2.5, and 3.2.8.

Principle 2 instructs us how to detect conditional independencies from the structure of the model, i.e., the graph. This principle states that, regardless of the functional form of the equations in a recursive model $M$, and regardless of the distribution of the exogenous variables $U$, if the disturbances are mutually independent, the distribution $P(v)$ of the endogenous variables must obey certain conditional independence relations, stated roughly as follows:

Whenever sets $X$ and $Y$ of nodes in the graph are "separated" by a set $Z, X$ is independent of $Y$ given $Z$ in the probability. ${ }^{17}$

\footnotetext{
${ }^{17}$ The "separation" criterion requires that all paths between $X$ and $Y$ be intercepted by $Z$, with special handling of paths containing head-to-head arrows (Pearl, 1993; Pearl, 2000, pp. 16-18). In linear models, Principle 2 is valid for non-recursive models as well.
} 
This powerful theorem, called $d$-separation (Pearl and Verma, 1987; Pearl, 2000, pp. 16-18; Verma and Pearl, 1990) constitutes the semantic link between the causal assumptions encoded in the model and the constraints which they induce on the observed data. The theorem permits all conditional independencies implied by a given model to be read off the graph, thus saving researchers the laborious effort of deriving such independencies algebraically. ${ }^{18}$ Because of this feature, the $d$ separation criterion serves as the basis for all modern approaches to causal inference, including causal discovery (Pearl and Verma, 1991; Spirtes et al., 1993) causal identification and misspecification testing.

\section{Remarks on the "Structuralists" vs. "Experimentalists" Debate}

The Spring 2010 issue of the Journal of Econometric Perspectives (Vol. 24, No. 2) presented an interesting discussion on causal inference between two camps of economists: the "structuralists" and the "experimentalists;" the former acknowledge their reliance on modelling assumptions, the latter argue that they don't, or claim to minimize such reliance. Angrist and Pischke (2010) represented the "experimentalist" position and Keane (2010), Leamer (2010), Nevo and Whinston (2010), and Sims (2010) defending the structural approach.

Viewed from the SCM perspective, the debate is rhetorical. We know, from first principles, that any causal conclusion drawn from observational studies must rest on untested causal assumptions. ${ }^{19}$ Therefore, whatever relation an instrumental design bears to an ideal controlled experiment is just one such assumption and, to the extent that the "experimental" approach is valid, it is a routine exercise in structural economics.

However, the philosophical basis of the "experimentalist" approach, as it is currently marketed, is both flawed and error prone. First, its sole reliance on instrumental variables weakens its inferential power and deprives researchers of other sources of information, not less reliable, which permit identification beyond linear models or LATE-type subpopulations. Second, and more importantly, the "experimentalist" paradigm takes similarities to the randomized experiment ideal to be its sole guiding principle, instead of harnessing all available knowledge, as well as Principle 1 and Principle 2 , towards answering the research question at hand. The fallibility of this paradigm has surfaced in a number of applications (e.g., Pearl 2009b, 2011c,b) and has given birth to a school of research that, in the name of mimicking controlled experiments avoids making modeling assumptions transparent. ${ }^{20}$

Another take on the "experimental - structural" debate is provided by Heckman (2010) who reiterates the superiority of the structural over the Neyman-Rubin model, but stops short of identifying the key element for that superiority. This is important because, after all, the structural and potential-outcome approaches are logically equivalent ${ }^{21}$ differing only in the languages used

\footnotetext{
${ }^{18}$ Heckman and Pinto (2013) propose to derive these independencies using the graphoid axioms (Dawid, 1979; Pearl and Paz, 1986; Pearl, 1988, pp. 82-115), a task requiring exponential complexity. The graphoid axioms are good for confirming a derivation (of one independence from others), but they are not very helpful in finding such derivation or in deciding whether one exists. DAGs, on the other hand, act as logical machines; they automatically compute all valid independencies and explicate them through simple path-separation conditions (Pearl and Verma, 1987).

${ }^{19}$ Cartwright (1989) named this principle "no causes in, no causes out," which follows formally from the theory of equivalent models (Verma and Pearl, 1990); for any model yielding a conclusion $C$, one can construct a statistically equivalent model that refutes $C$ and fits the data equally well.

${ }^{20}$ For example, one doctrine in this paradigm dictates that, because randomization balances pre-treatment covariances, the aim of the analysis should be to achieve such balance. This has led researchers to surmise that one should condition on all such covariates (Hirano and Imbens, 2001; Pearl, 2009b; Rubin, 2009). Another misguided doctrine denies causal character to non-manipulable variables, and has led to paradoxical mediation analysis using "principal strata" (Pearl, 2011b).

${ }^{21}$ The equivalence was shown in Galles and Pearl (1998) and Halpern (1998); a theorem in one is a theorem in the other, and an assumption in one has a corresponding assumption in the other. The two differ only in how substantive information is encoded. The potential outcome language insists on encoding such information in the form of conditional independence statements about counterfactual variables, a cognitively formidable task, while the structural equation model permits modelers to encode this information in the form of cause effect relationships representing economic mechanisms and processes. A simple translation between the two is given in (Pearl, 2000, pp. 231-234) which should bridge the wall between "experimentalists" and "structuralists." See Appendix 1 for a simple
} 
to encode assumptions; the former using equations, the latter using counterfactual independencies (see Pearl 2000, pp. 230-234). So why did the "experimentalists" end up with the primitive, single-equation exercises reported in Angrist and Pischke (2010)? Why did they not import the rich knowledge that structural modellers encode in their equations, to make their assumptions compelling, explicit and transparent?

The answer usually given is that "experimentalists" are a priori skeptical about the assumptions embedded in structural models, and feel more comfortable with those involved in instrumental variables design. However, since the very choice of an instrument rests on the type of modeling assumptions that "experimentalists" attempt to avoid, namely, exclusion and exogeneity (see Section 3.2.4), why did "experimentalists" embrace the former and reject the latter? Moreover, why did they exempt the former from explicit representation in the model, so that they can be reasoned about formally or examined for possible testable implications?

This practice in the "experimental" camp has also puzzled Sims (2010), who wrote: "using instrumental variable formulas while simply listing the instruments, with little or no discussion of what kind of larger multivariate system would justify isolating the single equation or small system to which the formulas are applied, was, and to some extent still is, a common practice."

I believe the reason for this practice lies not in mistrust of modeling assumptions but in mathematical ineptness to read those assumptions and derive their consequences, as dictated by the two principles described in Section 3.4. By rejecting structural equations as a language for expressing substantive economic knowledge, and confining themselves exclusively to the language of potential outcomes "experimentalists" have in effect cut themselves off from the one language in which large number of relationships can be expressed meaningfully and reasoned about.

This uncompromising rejection has also deprived "experimentalists" from acquiring the basic tools of identifying instrumental variables in a system of equations (3.2.4) or solving elementary problems such as those posed in 3.2. Risking errors and oversight (Pearl, 2009b), they have chosen to shun these tools for reasons ranging from "nonscientific ad hockery" (Rubin, 2010) to selective unawareness (Imbens and Wooldridge, 2009). It is not lack of good intention, but lack of modern mathematical tools that prevents the "experimentalists" from conducting a "discussion of what kind of larger multivariate system would justify" their formulas. ${ }^{22}$

\section{Conclusions}

This paper traces the logic and mathematical machinery needed for causal analysis from the original insights advanced by Haavelmo to the nonparametric analysis of Structural Causal Models (SCM). We have demonstrated by examples the type of queries the SCM framework can answer, the assumptions required, the language used for encoding those assumptions and the mathematical operations needed for deriving causal and counterfactual conclusions.

Not surprisingly, graphical formalism was found to be the most succinct, natural and effective language for representing nonparametric structural equations; it highlights the assumptions and abstracts away unnecessary algebraic details. It is for these reasons that graphical representations have become an indispensable second language in the health sciences (Glymour and Greenland, 2008; Vansteelandt and Lange, 2012) and are making their way towards the social and behavioral sciences (Chalak and White, 2011; Lee, 2012; Morgan and Winship, 2007). Recent adaptation of graphical methods by econometricians (Heckman and Pinto, 2013), albeit under the cover of criticism (Pearl, 2013b), further attests to their power and applicability. I am convinced therefore that, once the power of graphical tools is recognized through simple examples, economists too will add them to their arsenal of formal methods and be able to reap the benefits of causal analysis, parametric as well as nonparametric. ${ }^{23}$ Acquiring these tools would enable researchers to recognize the testable

\footnotetext{
illustration of the equivalence of the two notational systems.

${ }^{22}$ The potential outcome language, is rather inept for capturing substantive knowledge of the kind carried by structural equation models. The restricted vocabulary of "ignorability," "treatment assignment" and "missing data" that has ruled (and still rules) the potential-outcome paradigm is not flexible enough to specify transparently even the most elementary models (say a three-variable Markov chain) that researchers wish to hypothesize (Pearl, 2011a).

${ }^{23} \mathrm{~A}$ recent survey of econometric textbooks (Chen and Pearl, 2013) has somewhat tempered my optimism at the
} 
implications of a system of equations, locate instruments in such systems, decide if two such systems are equivalent, if causal effects are identifiable, if two counterfactuals are independent given another, whether a set of measurements will reduce bias, and, most importantly, reading the causal and counterfactual information that such systems convey.

The development of powerful mathematical tools for deriving or predicting the logical ramifications of untested theoretical assumptions will enable us to reverse-engineer our inferences and learn to minimize sensitivity to those assumptions.

\section{Acknowledgment}

This paper has benefited from discussions with J.H. Abbring, Elias Bareinboim, David Bessler, Bryant Chen, James Heckman, Kevin Hoover, Ed Leamer, Rosa Matzkin, Chris Sims, James Stock, and Hal White. I am particularly grateful to the Guest Editor, Dr. Olav Bjerkholt, for inviting me to participate in this centennial issue and for offering thoughtful and helpful comments on earlier versions of this paper.

This research was supported in parts by grants from NSF \#IIS-1249822 and \#IIS-1302448 and ONR \#N00014-13-1-0153 and \#N00014-10-1-0933.

\section{Appendix 1}

This Appendix lays out the conceptual and formal relationships between structural equation modeling (SEM) in economics and the potential outcome (PO) framework, usually associated with Neyman (1923) and Rubin (1974). Some researchers regard PO as an indispensable tool in modeling experiments and quasi-experiments in econometric studies (Angrist and Pischke, 2010; Imbens and Wooldridge, 2009). This Appendix shows that the PO framework and all its ramifications for experiments and quasi-experiments follow naturally from standard SEM, and the causal interpretation given to it by Haavelmo (1943).

Our starting point will be a typical structural equation

$$
y=g(x, u)
$$

in which $X$ and $U$ are arbitrary random variables, jointly distributed by a probability function $P(x, u)$, and $g$ an arbitrary function that maps $X$ and $U$ onto an "outcome" variable $Y$. Together, the three variables are jointly distributed by a probability function $P(x, y, u)$, of which only the marginal $P(x, y)=\sum_{u} P(x, y, u)$ can be estimated from sampled data. ${ }^{24}$

Variable $X$, sometimes called "treatment" or "independent variable" may represent a policy or an economic condition (e.g., education, income, prices, taxes, interest rates), whose effects are of interest and whose status agents may choose on their own (in non-experimental setting). Variable $U$, also called "disturbance," represents all other factors, mostly unobserved, that account for the variability of $Y$ when $X$ is held constant. The causal interpretation of structural equations regards Eq. (A.1) as a process by which Nature assigns values to $Y$ after consulting the values of $X$ and $U$.

Let us now define a counterfactual random variable $Y_{x}$ that represents "the value that $Y$ would attain if $X$ were $x$." According to Eq. (4), this variable is defined by:

$$
Y_{x}=g(x, U)
$$

where $x$ is a constant (usually $x=1,0$ ), and where the the disturbance term $U$ is governed by the

pace at which economists lift themselves to the age of modernity, as most surveyed textbooks were found to conflate regressional and structural vocabulary with stunning laxity. I hope, however, that this paper will entice concerned educators and authors to write "causal inference addenda" to supplement and illuminate standard econometric texts.

${ }^{24}$ Integrals should replace summations when continuous variables are involved. 
distribution $^{25}$

$$
P(U=u)=\sum_{x} P(x, u)
$$

Given these preliminaries we will now prove four assertions about $Y_{x}$ and its relations to $Y$ and $X$.

\section{Assertion-1}

If $X$ and $U$ are independent then, for any functional relation $y=g(x, u)$ and any $x$ in the support of $X$, we have

$$
P\left(Y_{x}=y\right)=P(Y=y \mid X=x)
$$

In other words, the distribution of the counterfactual $Y_{x}$ is identified from observations on $X$ and $Y$, and is given by the conditional probability of $Y$ given $X=x$.

As a corollary, we conclude that in a randomized trial, where $X$ and $U$ are independent, the average causal effect of $X$ on $Y$ is identified, and is given by the regression

$$
E\left(Y_{x^{\prime}}-Y_{x}\right)=E_{\exp }\left(Y \mid X=x^{\prime}\right)-E_{\exp }(Y \mid X=x)
$$

Here $E_{\text {exp }}$ designates expectation according to the experimental distribution, to be distinguished from $E$, which stands for expectation according to the pretreatment distribution $P(x, y)=\sum_{u} P(x, y, u)$.

\section{Assertion-2}

Regardless of how $X$ and $U$ are distributed, the following relationship holds between $X, Y$, and $Y_{x}$

$$
X=x \Longrightarrow Y_{x}=Y
$$

or, in case $X$ is binary,

$$
Y=x Y_{1}+(1-x) Y_{0}
$$

An immediate consequence of (A.6) is the equation

$$
P\left(Y_{x}=y \mid Z=z, X=x\right)=P(Y=y \mid Z=z, X=x)
$$

which holds for any sets of variables $X, Y$, and $Z$. It permits us to convert expressions involving probabilities of counterfactuals to expressions involving ordinary conditional probabilities of measured variables.

Eq. (A.6), also called "consistency rule," is treated as an extra assumption in the PO framework (Rubin, 1974), where it is used to insure the purity of the experiment (e.g., no side-effects of treatments). It asserts, for example, that a patient who recovered after taking treatment $X=x$ by choice would also have recovered if assigned treatment $X=x$ by design. In the SEM framework, in contrast, consistency is logically entailed by definition (A.2), and purity of experiments remains the responsibility of the experimenter (see footnote 6 and (Pearl, 2010c)).

\section{Assertion-3}

Regardless of how $X$ and $U$ are distributed, the slope, $\beta$, in the linear structural equation

$$
y=\alpha+\beta x+u
$$

\footnotetext{
${ }^{25}$ The invariance of (A.3) under the intervention $X=x$ follows from Eq. (4) which interprets the counterfactual as an incisive "surgery" that suppresses all mechanisms that may contribute to variations in $X$ and imposes the equality $X=x$ without perturbing $U$ or any other variable that is not affected by $X$. Such a "surgery" is not needed in our single-equation case, since $X$ is part of the equation for $Y$; enforcing $X=x$ suffices.
} 
is given by

$$
\beta=E\left(Y_{1}-Y_{0}\right)
$$

or, for non-binary $X$,

$$
\beta=E\left(Y_{x^{\prime}}-Y_{x}\right) /\left(x^{\prime}-x\right)
$$

\section{Assertion-4}

Exogeneity implies "strong ignorability." Formally,

$$
U \Perp X \Longrightarrow\left\{Y_{0}, Y_{1}\right\} \Perp X
$$

The independence on the left hand side expresses the standard econometric condition for exogeneity of $X$ (relative to the equation of $Y$ ), while the one on the right hand side is a distinctive creation of the PO framework, called "strong ignorability" (Rosenbaum and Rubin, 1983). Almost all inferences in the PO framework invoke this assumption or its "conditional ignorability" variant, and is often advertised as a more "principled" or more "explicit" assumption than its "exogeneity" counterpart (Angrist, Imbens, and Rubin, 1996). It is not. Even avid PO advocates resort to "omitted factors" when the need arises to defend or criticize the opaque assumption of "ignorability" (Pearl, 2000, Second Edition, pp. 341-344). Because of its opacity, "ignorability" is used primarily as a syntactic license for certain statistical routines, rather than a condition deserving justification (see footnote 22). ${ }^{26}$

Properties (A.4)-(A.7), which are normally attributed to potential outcome analysis, are here shown to emerge organically from standard structural modeling in economics. The latter provides, therefore, the scientific basis for the former, and extends counterfactual analysis beyond the experimental paradigm that constrains the PO framework.

\section{Proofs}

\section{Proof of Assertion-1}

We start with $P(Y=y \mid X=x)$ and, using the indicator function

$$
1(A)=\left\{\begin{array}{l}
1 \text { if } A \text { is true } \\
0 \text { if } A \text { is false }
\end{array}\right.
$$

we write:

$$
\begin{aligned}
P(Y=y \mid X=x) & =P(g(x, U)=y \mid X=x) \\
& =\sum_{u} 1(g(x, u)=y) P(U=u \mid X=x) \\
& =\sum_{u} 1(g(x, u)=y) P(u) \\
& =P(g(x, U)=y) \\
& =P\left(Y_{x}=y\right)
\end{aligned}
$$

\footnotetext{
${ }^{26}$ The role of potential outcomes in randomized trials is typically described as follows: "Because an individual's treatment status is randomly assigned, it is distributed independently of his or her potential outcomes" (Stock and Watson, 2011, p. 471). For this argument to hold, one needs to show first that the potential outcomes $\left\{Y_{1}, Y_{0}\right\}$ represent immutable characteristics of an individual that do not change with treatment status. There is nothing in the PO characterization of $\left\{Y_{1}, Y_{0}\right\}$ that compels this invariance and, hence, there is no a priori reason to assume that ignorability holds in randomized trials. This invariance follows in fact from the structural interpretation of potential outcomes according to which $\left\{Y_{1}, Y_{0}\right\}$ are none others but the factors included in $U$, and those are unaffected by $X$ a priori.
} 
which proves (A.4).

To prove Corollary (A.5) we note that, since a randomized control trial renders $X$ independent on $U$, the average causal effect of incrementing the treatment from $X=x$ to $X=x^{\prime}$ is given by

$$
E\left(Y_{x^{\prime}}-Y_{x}\right)=E_{\exp }\left(Y \mid X=x^{\prime}\right)-E_{\exp }(Y \mid X=x)
$$

\section{Proof of Assertion-2}

Implication (A.6) follows from the definition of $Y_{x}$, because under the condition $X=x$ the expression of $Y$ (A.1) and $Y_{x}$ (A.2) coincide. Expression (A.7) merely encodes this implication for binary $X$.

\section{Proof of Assertion-3}

(A.9) follows by substituting the function

$$
g(x, u)=\alpha+\beta x+u
$$

into the definitions of $Y_{1}$ and $Y_{0}$ (A.2), yielding

$$
\begin{aligned}
E\left(Y_{1}-Y_{0}\right) & =E[g(1, U)-g(0, U)] \\
& =E[\alpha+\beta \times 1+U-\alpha-\beta \times 0-U] \\
& =\beta
\end{aligned}
$$

\section{Proof of Assertion-4}

Since both $Y_{0}$ and $Y_{1}$ are deterministic functions of $U$ (see A.2), it is clear that if $U$ is independent of $X$ so is the joint variable $\left\{Y_{0}, Y_{1}\right\}$. This proves (A.10).

\section{References}

Angrist, J., Imbens, G. and Rubin, D. (1996). Identification of causal effects using instrumental variables (with comments). Journal of the American Statistical Association 91 444-472.

Angrist, J. D. and Pischke, J.-S. (2010). The credibility revolution in empirical economics: How better research design is taking the con out of econometrics. Journal of Economic Perspectives 24 3-30.

Balke, A. and Pearl, J. (1995). Counterfactuals and policy analysis in structural models. In Uncertainty in Artificial Intelligence 11 (P. Besnard and S. Hanks, eds.). Morgan Kaufmann, San Francisco, 11-18.

Balke, A. and Pearl, J. (1997). Bounds on treatment effects from studies with imperfect compliance. Journal of the American Statistical Association 92 1172-1176.

Bareinboim, E. and Pearl, J. (2012). Controlling selection bias in causal inference. In Proceedings of the 15th International Conference on Artificial Intelligence and Statistics (AISTATS) (N. Lawrence and M. Girolami, eds.). JMLR, La Palma, Canary Islands, 100-108.

Bareinboim, E., Tian, J. and Pearl, J. (2014). Recovering from selection bias in causal and statistical inference. Tech. Rep. R-425, <http://ftp.cs.ucla.edu/pub/stat_ser/r425.pdf >, Department of Computer Science, University of California, Los Angeles, CA. Forthcoming Proceedings of the Twenty-Eighth Conference on Artificial Intelligence (AAAI-14), 2014. Available ?at http://ftp.cs.ucla.edu/pub/stat_ser/r425.pdf. 
Brito, C. and Pearl, J. (2002). Generalized instrumental variables. In Uncertainty in Artificial Intelligence, Proceedings of the Eighteenth Conference (A. Darwiche and N. Friedman, eds.). Morgan Kaufmann, San Francisco, 85-93.

Brito, C. and Pearl, J. (2006). Graphical condition for identification in recursive SEM. In Proceedings of the Twenty-Third Conference on Uncertainty in Artificial Intelligence. AUAI Press, Corvallis, OR, 47-54.

Campbell, D. and Stanley, J. (1963). Experimental and Quasi-Experimental Designs for Research. Wadsworth Publishing, Chicago.

Cartwright, N. (1989). Nature's Capacities and Their Measurement. Clarendon Press, Oxford.

Cartwright, N. (2007). Hunting Causes and Using Them: Approaches in Philosophy and Economics. Cambridge University Press, New York, NY.

Chalak, K. and White, H. (2011). An extended class of instrumental variables for the estimation of causal effects. Canadian Journal of Economics 44 1-31.

Chen, B. and Pearl, J. (2013). Regression and causation: A critical examination of econometrics textbooks. Real-World Economics Review 65 2-20.

DAwid, A. (1979). Conditional independence in statistical theory. Journal of the Royal Statistical Society, Series B 41 1-31.

Engle, R., Hendry, D. and Richard, J. (1983). Exogeneity. Econometrica 51 277-304.

Foygel, R., Draisma, J. and Drton, M. (2012). Half-trek criterion for generic identifiability of linear structural equation models. The Annals of Statistics 40 1682-1713.

Galles, D. and Pearl, J. (1998). An axiomatic characterization of causal counterfactuals. Foundation of Science 3 151-182.

Glymour, M. and Greenland, S. (2008). Causal diagrams. In Modern Epidemiology (K. Rothman, S. Greenland and T. Lash, eds.), 3rd ed. Lippincott Williams \& Wilkins, Philadelphia, PA, 183-209.

Goldberger, A. (1992). Models of substance; comment on N. Wermuth, 'On block-recursive linear regression equations'. Brazilian Journal of Probability and Statistics 6 1-56.

Greenland, S. and Pearl, J. (2011). Adjustments and their consequences - collapsibility analysis using graphical models. International Statistical Review 79 401-426.

HaAvelmo, T. (1943). The statistical implications of a system of simultaneous equations. Econometrica 11 1-12. Reprinted in D.F. Hendry and M.S. Morgan (Eds.), The Foundations of Econometric Analysis, Cambridge University Press, 477-490, 1995.

HaAvelmo, T. (1944). The probability approach in econometrics (1944). Supplement to Econometrica 12 12-17, 26-31, 33-39.

Halpern, J. (1998). Axiomatizing causal reasoning. In Uncertainty in Artificial Intelligence (G. Cooper and S. Moral, eds.). Morgan Kaufmann, San Francisco, CA, 202-210. Also, Journal of Artificial Intelligence Research 12 17-37, 2000.

Heckman, J. (1979). Sample selection bias as a specification error. Econometrica 47 153-161.

Heckman, J. (2000). Causal parameters and policy analysis in economics: A twentieth century retrospective. The Quarterly Journal of Economics 115 45-97.

Heckman, J. (2003). Conditioning causality and policy analysis. Journal of Econometrics 112 $73-78$. 
Heckman, J. (2008). Econometric causality. International Statistical Review 76 1-27.

HECKMAN, J. (2010). Building bridges between structural and program evaluation approaches to evaluating policy. Journal of Economic Literature 48 356-398.

Heckman, J. and Pinto, R. (2013). Causal analysis after Haavelmo. Tech. Rep. NBER Technical Working Paper 19453, National Bureau of Economic Research, MA.

Heckman, J. and Vytlacil, E. (2007). Handbook of Econometrics, vol. 6B, chap. Econometric Evaluation of Social Programs, Part I: Causal Models, Structural Models and Econometric Policy Evaluation. Elsevier B.V., Amsterdam, 4779-4874.

Hirano, K. and Imbens, G. (2001). Estimation of causal effects using propensity score weighting: an application to data on right heart catheterization. Health Services and Outcomes Research Methodology 2 259-278.

Hoover, K. D. (2011). Counterfactuals and causal structure. In Causality in the Sciences (P. M. Illari, F. Russo and J. Williamson, eds.). Clarendon Press, Oxford, 338-360.

Imbens, G. and Wooldridge, J. (2009). Recent developments in the econometrics of program evaluation. Journal of Economic Literature 47 5-86.

Keane, M. P. (2010). A structural perspective on the experimentalist school. Journal of Economic Perspectives 24 47-58.

Kyono, T. (2010). Commentator: A front-end user-interface module for graphical and structural equation modeling. Tech. Rep. R-364, <http://ftp.cs.ucla.edu/pub/stat_ser/r364.pdf $>$, Master Thesis, Department of Computer Science, University of California, Los Angeles, CA.

Leamer, E. E. (2010). Tantalus on the road to asymptopia. Journal of Economic Perspectives 24 $31-46$.

LEE, J. J. (2012). Correlation and causation in the study of personality. European Journal of Personality 26 372-390.

LEwis, D. (1973). Counterfactuals. Harvard University Press, Cambridge, MA.

LuCAS JR., R. (1976). Econometric policy evaluation: A critique. In The Phillips Curve and Labor Markets (K. Brunner and A. Meltzer, eds.), vol. CRCS, Vol. 1. North-Holland, Amsterdam, 19-46.

MARSChaK, J. (1953). Economic measurements for policy and prediction. In Studies in Econometric Method (W. C. Hood and T. Koopmans, eds.). Cowles Commission Monograph 10, Wiley and Sons, Inc., 1-26.

Morgan, S. and Winship, C. (2007). Counterfactuals and Causal Inference: Methods and Principles for Social Research (Analytical Methods for Social Research). Cambridge University Press, New York, NY.

Nevo, A. and Whinston, M. D. (2010). Taking the dogma out of econometrics: Structural modeling and credible inference. Journal of Economic Perspectives 24 69-82.

Neyman, J. (1923). Sur les applications de la thar des probabilities aux experiences Agaricales: Essay des principle. English translation of excerpts by D. Dabrowska and T. Speed (1990), in Statistical Science 5 463-472.

Oster, E. (2013). Unobservable selection and coefficient stability: Theory and validation. Tech. Rep. No. 19054, National Bureau of Economic Research, Cambridge, MA.

Pearl, J. (1988). Probabilistic Reasoning in Intelligent Systems. Morgan Kaufmann, San Mateo, CA. 
Pearl, J. (1993). Comment: Graphical models, causality, and intervention. Statistical Science 8 $266-269$.

Pearl, J. (1994). A probabilistic calculus of actions. In Uncertainty in Artificial Intelligence 10 (R. L. de Mantaras and D. Poole, eds.). Morgan Kaufmann, San Mateo, CA, 454-462.

Pearl, J. (2000). Causality: Models, Reasoning, and Inference. Cambridge University Press, New York. 2nd edition, 2009.

Pearl, J. (2001). Direct and indirect effects. In Proceedings of the Seventeenth Conference on Uncertainty in Artificial Intelligence. Morgan Kaufmann, San Francisco, CA, 411-420.

Pearl, J. (2004). Robustness of causal claims. In Proceedings of the Twentieth Conference Uncertainty in Artificial Intelligence (M. Chickering and J. Halpern, eds.). AUAI Press, Arlington, VA, 446-453.

Pearl, J. (2009a). Causality: Models, Reasoning, and Inference. 2nd ed. Cambridge University Press, New York.

Pearl, J. (2009b). Myth, confusion, and science in causal analysis. Tech. Rep. R-348, University of California, Los Angeles, CA. <http://ftp.cs.ucla.edu/pub/stat_ser/r348.pdf>.

Pearl, J. (2010a). The foundations of causal inference. Sociological Methodology 40 75-149.

PEARL, J. (2010b). An introduction to causal inference. The International Journal of Biostatistics 6 Issue 2, Article 7 DOI: 10.2202/1557-4679.1203. Available at: $<$ http://ftp.cs.ucla.edu/pub/stat_ser/r354-corrected-reprint.pdf $>$.

Pearl, J. (2010c). On the consistency rule in causal inference: Axiom, definition, assumption, or theorem? Epidemiology 21 872-875.

Pearl, J. (2010d). Review of N. Cartwright 'Hunting causes and using them'. Economics and Philosophy 26 69-77.

Pearl, J. (2011a). Graphical models, potential outcomes and causal inference: Comment on Lindquist and Sobel. Statistics in Medicine 58 770-771.

Pearl, J. (2011b). Principal stratification a goal or a tool? The International Journal of Biostatistics 7, Issue 1, Article 20. DOI: 10.2202/1557-4679.1322. Available at: $<$ http://ftp.cs.ucla.edu/pub/stat_ser/r382.pdf $>$.

Pearl, J. (2011c). Understanding bias amplification. American Journal of Epidemiology 174 1223-1227. DOI: 10.1093/aje/kwr352.

Pearl, J. (2012a). The causal foundations of structural equation modeling. In Handbook of Structural Equation Modeling (R. Hoyle, ed.). Guilford Press, New York, 68-91.

Pearl, J. (2012b). The causal mediation formula - a guide to the assessment of pathways and mechanisms. Prevention Science 13 426-436, DOI: 10.1007/s11121-011-0270-1.

Pearl, J. (2013a). Linear models: A useful "microscope" for causal analysis. Journal of Causal Inference 1 155-170.

Pearl, J. (2013b). Reflections on Heckman and Pinto's 'causal analysis after Haavelmo'. Tech. Rep. R-420, <http://ftp.cs.ucla.edu/pub/stat_ser/r420.pdf>, Department of Computer Science, University of California, Los Angeles, CA. Working paper.

Pearl, J. (2014). Interpretation and identification of causal mediation. Tech. Rep. R-389, $<$ http://ftp.cs.ucla.edu/pub/stat_ser/r389.pdf $>$, Department of Computer Science, University of California, Los Angeles, CA. Forthcoming Psychological Methods, (2014). 
Pearl, J. and Bareinboim, E. (2011). Transportability of causal and statistical relations: A formal approach. In Proceedings of the Twenty-Fifth Conference on Artificial Intelligence (AAAI-11). Menlo Park, CA. Available at: <http://ftp.cs.ucla.edu/pub/stat_ser/r372a.pdf>.

Pearl, J. and PAz, A. (1986). On the logic of representing dependencies by graphs. In Proceedings of the Canadian AI Conference. Montreal, Ontario, Canada, 94-98.

Pearl, J. and Verma, T. (1987). The logic of representing dependencies by directed graphs. In Proceedings of the Sixth National Conference on Artificial Intelligence. Morgan Kaufmann Publishers, Los Altos, CA, 374-379.

Pearl, J. and Verma, T. (1991). A theory of inferred causation. In Principles of Knowledge Representation and Reasoning: Proceedings of the Second International Conference (J. Allen, R. Fikes and E. Sandewall, eds.). Morgan Kaufmann, San Mateo, CA, 441-452.

Phiromswad, P. and Hoover, K. D. (2013). Selecting instrumental variables: A graph-theoretic approach. Available at SSRN: http://ssrn.com/abstract=2318552 or http://dx.doi.org/10.2139/ssrn.2318552.

Richard, J. (1980). Models with several regimes and changes in exogeneity. Review of Economic Studies 47 1-20.

Rosenbaum, P. and Rubin, D. (1983). The central role of propensity score in observational studies for causal effects. Biometrika $7041-55$.

RuBin, D. (1974). Estimating causal effects of treatments in randomized and nonrandomized studies. Journal of Educational Psychology 66 688-701.

Rubin, D. (2009). Author's reply: Should observational studies be designed to allow lack of balance in covariate distributions across treatment group? Statistics in Medicine 28 1420-1423.

Rubin, D. (2010). Reflections stimulated by the comments of Shadish (2010) and West and Thoemmes (2010). Psychological Methods 15 39-46.

Shpitser, I. and PeArl, J. (2008). Complete identification methods for the causal hierarchy. Journal of Machine Learning Research 9 1941-1979.

Simon, H. and Rescher, N. (1966). Cause and counterfactual. Philosophy and Science 33 323-340.

Sims, C. A. (2010). But economics is not an experimental science. Journal of Economic Perspectives $2459-68$.

Spanos, A. (2010). Theory testing in economics and the error-statistical perspective. In Error and Inference (D. G. Mayo and A. Spanos, eds.). Cambridge University Press, New York, 202-246.

Spirtes, P., Glymour, C. and Scheines, R. (1993). Causation, Prediction, and Search. SpringerVerlag, New York.

Stock, J. and Watson, M. (2011). Introduction to Econometrics. 3rd ed. Addison-Wesley, New York.

Strotz, R. and Wold, H. (1960). Recursive versus nonrecursive systems: An attempt at synthesis. Econometrica 28 417-427.

VAnsteelandt, S. and Lange, C. (2012). Causation and causal inference for genetic effects. Human Genetics, (Special Issue on Genetic Epidemiology: Study Designs and Methods PostGWAS) 131 1665-1676. 
Verma, T. and Pearl, J. (1990). Equivalence and synthesis of causal models. In Proceedings of the Sixth Conference on Uncertainty in Artificial Intelligence. Cambridge, MA. Also in P. Bonissone, M. Henrion, L.N. Kanal and J.F. Lemmer (Eds.), Uncertainty in Artificial Intelligence 6, Elsevier Science Publishers, B.V., 255-268, 1991.

Wermuth, N. (1992). On block-recursive regression equations. Brazilian Journal of Probability and Statistics (with discussion) 6 1-56.

White, H. and Lu, X. (2014). Robustness checks and robustness tests in applied economics. Journal of Econometrics 178 194-206. 